\title{
Emerging Technologies for Use in the Study, Diagnosis, and Treatment of Patients with COVID-19
}

\author{
Maria Tsikala Vafea, Eleftheria Atalla, Joanna Georgakas, Fadi Shehadeh, \\ Evangelia K. Mylona, Markos Kalligeros, and Eleftherios Mylonakis \\ Infectious Diseases Division, Rhode Island Hospital, Warren Alpert Medical School of Brown University, 593 Eddy Street, POB, \\ 3rd Floor, Suite 328/330, Providence, RI 02903, USA
}

(Received 28 May 2020; accepted 18 June 2020; published online 24 June 2020)

Associate Editor Michael R. King oversaw the review of this article.

\begin{abstract}
Introduction-The COVID-19 pandemic has caused an unprecedented health and economic worldwide crisis. Innovative solutions are imperative given limited resources and immediate need for medical supplies, healthcare support and treatments.

Aim - The purpose of this review is to summarize emerging technologies being implemented in the study, diagnosis, and treatment of COVID-19.

Results - Key focus areas include the applications of artificial intelligence, the use of Big Data and Internet of Things, the importance of mathematical modeling for predictions, utilization of technology for community screening, the use of nanotechnology for treatment and vaccine development, the utility of telemedicine, the implementation of 3D-printing to manage new demands and the potential of robotics.

Conclusion - The review concludes by highlighting the need for collaboration in the scientific community with open sharing of knowledge, tools, and expertise.
\end{abstract}

Keywords-Artificial intelligence, Nanomedicine, Vaccines, Drug repurposing, Monoclonal antibodies, Mathematical modeling, Big Data, Internet of Things, Community screening, Telemedicine, Robots, 3D-printing.

\section{ABBREVIATIONS}

3D 3-Dimensional

ACE Angiotensin-Converting Enzyme

AI Artificial Intelligence

Address correspondence to Eleftherios Mylonakis, Infectious Diseases Division, Rhode Island Hospital, Warren Alpert Medical School of Brown University, 593 Eddy Street, POB, 3rd Floor, Suite 328/330, Providence, RI 02903, USA. Electronic mail: emylonakis@lifespan.org

Maria Tsikala Vafea and Eleftheria Atalla contributed equally to this work.

$\begin{array}{ll}\text { ARDS } & \text { Acute Respiratory Distress Syndrome } \\ \text { CDC } & \begin{array}{l}\text { Centers for Disease Control and Preven- } \\ \text { tion }\end{array} \\ \text { CNN } & \text { Convolutional Neural Networks } \\ \text { CT } & \text { Computed Tomography } \\ \text { GPS } & \text { Global Positioning System } \\ \text { HA } & \text { Hemagglutinin } \\ \text { HIV } & \text { Human Immunodeficiency Virus } \\ \text { IBM } & \text { Individual Based Models } \\ \text { IoT } & \text { Internet of Things } \\ \text { IoMT } & \text { Internet of Medical Things } \\ \text { MRI } & \text { Magnetic Resonance Imaging } \\ \text { NHIA } & \text { National Health Insurance Administration } \\ \text { NIA } & \text { National Immigration Agency } \\ \text { NP } & \text { Nanoparticles } \\ \text { PPE } & \text { Personal Protective Equipment } \\ \text { RBD } & \text { Receptor Binding Domain } \\ \text { S } & \text { Spike } \\ \text { SEIR } & \text { Susceptible-Exposed-Infected-Recovered } \\ \text { SIR } & \text { Susceptible-Infected-Recovered } \\ \text { UAV } & \text { Unmanned Aerial Vehicles } \\ \text { UV } & \text { Ultraviolet }\end{array}$

\section{INTRODUCTION}

A novel coronavirus (SARS-CoV-2, causing the disease COVID-19), was first identified in the city of Wuhan, in Hubei Province, China in December 2019 and has rapidly spread across the globe. ${ }^{60}$ The COVID-19 pandemic has caused an unprecedented health and economic crisis. The magnitude of the crisis has called for an immediate response from governing officials, scientists, and medical professionals. With 
limited resources and immediate need for medical supplies, healthcare support and treatments, the crisis has demanded innovative solutions. Novel uses of emerging technologies have been proposed in order to meet the rising demands.

Emerging technologies have aided in the study of COVID-19, the development of advanced diagnostic tools and treatments, and the response to medical supply shortages. The innovative use of emerging technologies continues to have a profound impact on our ability to respond to this global crisis and should continue to be utilized to help improve outcomes. The aim of this review is to describe the features and uses of new technological developments implemented to combat the COVID-19 pandemic. The technologies in this review include: artificial intelligence (AI), machine learning and deep learning, nanomedicine, novel technologies for vaccines development and therapeutics, novel mathematical modeling, big data, internet of things (IoT), telemedicine, robots, and 3D printing technology.

\section{EMERGING TECHNOLOGIES}

\section{Artificial Intelligence (AI)}

AI has a wide array of features and applications that can be implemented to aid our response to COVID-19. Researchers have used both machine learning and deep learning models to study, diagnose and treat COVID-19. Machine learning tools enable the study of large datasets of viral genomes and can therefore increase our foundational knowledge of COVID-19. Scientists utilized machine learning based models along with intrinsic genomic signatures to provide a timely and accurate taxonomic classification of the novel coronavirus sequences and to identify the potential origin. ${ }^{45}$ Containment of the pandemic relies significantly on early and accurate diagnosis. Mei et al proposed an AI system based on machine learning and deep learning models that combines demographic (age, sex) and clinical information (laboratory test results, reported symptoms, history of exposure etc.) with chest imaging findings for rapid identification of patients with COVID-19. ${ }^{35}$ Major limitations of the proposed model for its adoption into clinical practice are the lack of generalizability to other patient populations and the difficulty on model training. ${ }^{35}$ Imaging modalities has served as a valuable tool for detecting COVID-19, evaluating complications, and monitoring disease course. During the last years, AI has empowered conventional imaging approaches, including CT scans and X-rays, to meet the increasing challenges by providing detection accuracy and reliability. ${ }^{19}$ Re- cently, deep learning models, the core algorithms of $\mathrm{AI}$, have been used to develop a thoracic CT image analysis system, which can automatically detect COVID-19 patients and quantify the disease burden. ${ }^{16}$ Also, through volumetric measurement of the opacities burden, the AI-based image analysis tool allows for tracking of patient's disease progression. ${ }^{16}$

Application of an independent AI software system decreased the need of radiologists' annotations of the lesions in CT images, lifting a significant burden from frontline medical staff. ${ }^{65}$ The above contribution of deep learning technology highlights the impact AI can potentially have during a pandemic, where workflow heavily relies on human labor and shortages of human resources are a common problem.

Deep convolutional neural networks (CNN), a widely used deep learning architecture, enabled distinguishing between COVID-19 and other causes of pneumonia through chest X-ray image analysis. ${ }^{37}$ Investigators used a neural network which was designed to learn COVID-19 radiological pneumonia pattern and could yield accurate diagnostic results in a small cohort of infected patients. ${ }^{37}$ In addition to offering diagnostic accuracy and speed, AI can help reduce occupational exposure to the virus. Frontline healthcare practitioners, including CT and MRI technicians, are at high risk for contracting the virus given their frequent exposure to COVID-19 patients. Researchers proposed the use of a contactless workflow that can serve to protect the frontline workers and ensures that services continue to have adequate staffing. ${ }^{49}$ A visual AI empowered mobile CT platform allows the technician to determine the optimal scanning parameters such as identifying the pose and shape of the patient, and monitor the imaging procedure safely and efficiently. ${ }^{50}$

AI applications can also be utilized to predict the outcome of COVID-19 infections. Machine learning was implemented for development of a prognostic algorithm to estimate the mortality risk of a person with COVID-19. ${ }^{62}$ The prediction model was able to predict and help with the early identification of critically ill patients and through extraction of three key laboratory parameters: lactic dehydrogenase, lymphocyte count, and high-sensitivity C-reactive protein. ${ }^{62}$ The demand for intensive care unit-level care and ventilatory support provision has increased as a result of the current pandemic. The recognition of critical cases preemptively would enable appropriate evaluation of future demand, and thus allow institutions to be more prepared to react appropriately.

An AI empowered model can predict the development of high mortality complications of COVID-19, such as acute respiratory distress syndrome (ARDS). ${ }^{24}$ Researchers utilized such a model to identify features 
of initial disease presentation that are associated with later development of ARDS; these features included a mildly elevated alanine aminotransferase, the presence of myalgias and an elevated hemoglobin. ${ }^{24}$ Emerging AI-based methods can potentiate epidemiological models for real-time forecasting of disease trends. Modified auto-encoders, helped scientists predict the length of the progression and the transmission dynamics of the pandemic. ${ }^{21}$ An accurate forecasting method aids in the development of effective healthcare policies and helps address public health issues that have arisen with the COVID-19 pandemic.

Ongoing efforts have been made to implement AI in the development of effective therapeutic strategies against COVID-19. Machine learning principles can be applied to predict whether commercially approved medications can be used for disease treatment. ${ }^{31}$ This approach facilitates the repurposing of existing therapies in a time-efficient manner. A learning-based drug discovery model was also launched for the generation of a potential coronavirus main protease inhibitor. ${ }^{3}$ Playing a central role in the viral replication, the main protease seems to be an attractive target for the new therapies. $^{3}$

Despite the potential of AI to be implemented in the fight against COVID-19, AI systems are still at a preliminary stage. ${ }^{38}$ A rigorous quality control to test their validity and to ensure patient benefit and safety is warranted. ${ }^{25}$ Also, given the fact that AI algorithms need access to massive datasets, ethical concerns about patient privacy further hamper the clinical world adoption of AI tools in the healthcare setting. ${ }^{38}$

\section{Nanomedicine}

Chloroquine is an example of a medication that has been studied within this field. Chloroquine has inhibitory effects on the uptake of nanoparticles from cells. ${ }^{20}$ Specifically, studies have found clinically significant doses of the medication are associated with reduced accumulation of synthetic nanoparticles (NP) in cell lines and the mononuclear phagocyte system of mice. $^{3}$ Given the similarities in size and shape of the synthetic NP and SARS-CoV-2, nanoparticles can help in investigation for drug repurposing for COVID-19. Researchers designed NP-based peptides that mimic the virus-binding domain of ACE protein. ${ }^{17}$ This particular domain facilitates viral entry into a cell; thus, a NP mimetic could serve as an antagonist, inhibiting the virus from entering cells. Investigators have postulated that inhalers containing the mimetic would be effective in preventing virus activation in the lungs. ${ }^{17}$ Based on the association of COVID-19 severity with the inflammatory response, ${ }^{36}$ scientists are developing nanoparticles that can help in inflam- mation control. ${ }^{14}$ Lipid-based nanoparticles, loaded with immunomodulating and antioxidant molecules (adenosine and $\alpha$-tocopherol respectively), can selectively deliver their therapeutic cargo to the sites of acute inflammation and therefore modulate oxidation stress and cytokine response. ${ }^{14}$ Also, the use of peptide-based nanoparticles has been evaluated for the design of a SARS-CoV-2 vaccine. Self-assembling peptide nanoparticles that were prepared from SARSCov-1 spike (S) protein, successfully induced neutralizing antibodies against the coronavirus, subsequently preventing infection of Vero cells. ${ }^{43}$

\section{Novel Vaccine Development}

New technologies have enabled a possible COVID19 vaccine to be designed within just a few months of the initial outbreak. ${ }^{30}$ Vaccine technology platforms under development include nucleic acid, recombinant viral vector (replicating and non-replicating), and protein-based (subunits or virus-like particles). ${ }^{29}$

Advanced nucleic acid vaccine platforms are being investigated for SARS-CoV-2. The naked viral DNA enters the cell through electroporation, a technique that creates pores in cell membranes to increase nucleic acid uptake, and produces copies of S protein, ${ }^{9}$ the viral component that induces a neutralizing humoral immune response. ${ }^{58}$ Messenger RNA (mRNA) technology is another approach for SARS-CoV-2 vaccine development. Use of mRNA, the intermediary molecule that cells use to translate genetic information into proteins, is an alternative to employing DNA. mRNAbased vaccines are designed to contain all of the necessary viral genetic information, allowing the virus to produce proteins-antigens of interest within the host cell, thus mimicking a natural infection. ${ }^{41}$ The use of mRNA technology for vaccine development is promising, allowing for rapid vaccine production at a low cost. ${ }^{41}$ However, nucleic acid-based vaccines are not licensed for humans and thus their immunogenicity is not yet proven. ${ }^{9}$

In recombinant viral vectors vaccine approach, the SARS-CoV-2 S protein gene would be inserted in the genome of another virus, such as adenovirus or measles. These genetically engineered viruses can then deliver the genetic information for the production of SARS-CoV-2 proteins to the host, enabling an immune response. ${ }^{9}$ A novel vaccine candidate based on nonreplicating adenovirus vector $5(\mathrm{Ad} 5-\mathrm{nCoV})$ carrying the full-length $\mathrm{S}$ protein gene, has already entered phase II trials. ${ }^{26}$ Viral vector vaccines, such those using weakened measles, are also a possible approach to developing a SARS-CoV-2 vaccine. The replication of viral vectors mimics a natural infection. As the vectors replicate within the infected cells, a complete immune 
response, including both innate and humoral immunity, is triggered. , $^{47}$

A protein-based vaccines platform under evaluation, includes the direct injection of viral protein subunits, mainly $\mathrm{S}$ protein or a part of it called receptor binding domain (RBD). ${ }^{9} \mathrm{RBD}$ is a key component of $\mathrm{S}$ protein useful in viral attachment and membrane fusion. ${ }^{13}$ However, addition of vaccine adjuvants as well as multiple booster injections might be required for an adequate immune response. ${ }^{9}$ Virus-like particles, another protein-based vaccine approach, are molecules that closely resemble viruses but lack genetic material and therefore are not infectious. ${ }^{9}$ Despite the potential to induce a strong immune response such vaccines are harder to produce. ${ }^{9}$

A glycoprotein-based approach to vaccine development has also been explored. This approach targets viral glycosylation of the $\mathrm{S}$ protein. Glycosylation of viral spikes masks the immunogenic sites that are otherwise recognized by the host immune system. ${ }^{29}$ As such, glycosylation allows the virus to evade the host immune system. Many viruses are known to use this process. ${ }^{58}$ Investigators studying the glycan profile of SARS-CoV-2, however, found that the glycan density of spikes is not high enough to effectively shield the virus from the host immune defenses. ${ }^{58}$ This vulnerability in virus glycan shield may be beneficial for induction of potent neutralizing antibodies and therefore be implicated in vaccine design. ${ }^{58}$

\section{Monoclonal Antibodies}

Powerful technological platforms are used to determine if existing therapeutic modalities approved for other indications can also be efficacious in the treatment of COVID-19. ${ }^{12}$ Computer-aided drug repurposing tools have identified several monoclonal antibodies that have potential to treat COVID-19. ${ }^{12}$ Monoclonal antibodies have not traditionally been used for infectious disease management but due to in silico drug repurposing, they seem to be a promising addition to the therapeutic arsenal against COVID$19 .^{12}$

Novel coronavirus infection is characterized by a fulminant and fatal hyperinflammatory state, responsible for lung damage and multiorgan collapse. ${ }^{34}$ Interleukin-6 (IL-6) is considered to be a major driver of the cytokine storm and a predictor of fatality according to a recent study from China. ${ }^{34}$ Antibodies directly targeting the IL-6 receptor could theoretically suppress the overactivated immune system and optimize clinical outcomes. Tocilizumab, a monoclonal antibody against IL-6 receptor, is a licensed regimen for rheumatoid arthritis and cytokine release syndrome and recently approved for the treatment of COVID-
19. ${ }^{34}$ While the clinical trials for this treatment are still under investigation, a report of 21 patients who have undergone treatment suggests that tocilizumab is welltolerated and associated with rapid resolution of clinical symptoms and improved outcomes. ${ }^{61}$ Other FDA approved immunomodulatory medications are being investigated for potential use in the treatment of COVID-19 include: sarilumab (IL-6 receptor antagonist), bevacizumab (anti-vascular endothelial growth factor medication), eculizumab (antibody inhibiting terminal complement), and ulinastatin (tryspin inhibitor). ${ }^{48}$ In addition to monoclonal antibodies targeting key components of the immune response, monoclonal antibodies targeting the virus are also under development. Researchers identified a human monoclonal antibody that targets an epitope that is conserved in both SARS-CoV and SARS-CoV-2, and neutralizes the virus in cultured cells. ${ }^{56}$ Such neutralizing antibodies can support viral clearance and help in post-exposure prophylaxis. ${ }^{56}$ Also, due to the crossneutralizing properties, the antibody might be useful for potential future outbreaks caused by related coronaviruses. ${ }^{56}$

\section{Mathematical Modeling}

Mathematical modeling has been at the forefront of predicting COVID-19 transmission rates. These models have been essential for informed public policy decision-making that has prevented further spread of the virus. Mathematical models comprise of a set of equations that mimic reality and can be refined to include new information about the virus. From simple to more complex mathematical models, researchers around the world are trying to capture the interplay between a plethora of factors, ranging from micropathogens and individual or population interactions, to macro-scale environmental, socio-economic and demographic conditions. That interplay is essential in order to explore possible scenarios of the epidemic spread. These generated scenarios are used to guide outbreak control strategies and prevention policies. $^{7,42,54}$

Many different types of models have been implemented. Stochastic individual based models (IBM), as well as classic deterministic "susceptible-infected-recovered" (SIR) and "susceptible-exposed-infected-recovered" (SEIR) models are examples of models that have been used historically for different infectious diseases. New models like the "Stereographic Brownian Diffusion Epidemiology Model" are being developed during the COVID-19 pandemic in an effort to better explain the virus spread. ${ }^{7,42,54}$ The interpretation of these models is of the utmost importance. Given the mantra "mathematical models are as good as the data 
they use," different models should be viewed as complementary rather than searching for a singular correct model that can answer all questions. ${ }^{40}$ Furthermore, presenting model results alone is not enough. Given the necessity for rapid responses, scientists should rapidly and openly share their codes, so results can be replicated, evaluated, and improved. ${ }^{6}$

It should be noted, that despite their usefulness, mathematical models in the COVID-19 pandemic have also generated some concerns. Significant information about the transmission of the virus remains unknown, thus limiting the precision of forecasts. ${ }^{2}$ As results of modeling projections are communicated online, they are over-interpreted as anticipated potentials. As a result, dynamic models developed with the known limitations of theoretical plausibility and mathematical probabilities, are misinterpreted as evidence of what is actually going to happen. ${ }^{46}$

\section{Big Data}

With the creation of Big Data for analytics a wealth of information from around the world can be made available to scientists, doctors, epidemiologists and policymakers. Big Data has proven to be a useful tool for rapid real-time evaluations as a result. ${ }^{57} \mathrm{Big}$ Data Analytics has potential to play a key role in preventing COVID-19 related hospital outbreaks. The storage and provision of accurate personal travel and contact history allows disease tracing and screening to be conducted for all patients and visitors before they enter a medical facility. In Taiwan, the government has integrated and analyzed several Big Data from National Health Insurance Administration (NHIA), National Immigration Agency (NIA) and Taiwan Centers for Disease Control. This has provided real-time information for outdoor quarantine station of each hospital and clinic in the state. As a result, all visitors are screened via personal identification cards and any suspected carrier is further examined before entering the building. ${ }^{11}$ This model could serve as way to further limit disease transmission.

This same framework can be applied to allow for faster immigration clearance at airports and sea borders. For example, in Taiwan, the NHIA and the NIA launched the Entry Quarantine System. This system uses the past 14-day travel history of an individual and their NHIA identification card data to screen them for COVID-19. Travel history data is created at the time of departure or arrival at a Taiwan Airport. At this time travelers are required to complete a health declaration form by scanning a QR code. A mobile health declaration pass is then sent via SMS to phones using a local telecom operator. This system has allowed for faster immigration clearance for those with minimal risk. $^{57}$

\section{Internet of Things}

The Internet of Things (IoT) is a system of interconnected computing devices that can transfer data over a network without human involvement. ${ }^{51}$ In typical daily functioning, examples of IoT applications include home security systems and smart lighting arrangements which are controllable through smartphones. ${ }^{51}$ The Internet of Medical Things (IoMT) is the healthcare-specific version of IoT and can be implemented to provide relief to medical staff, ensure quarantine implementation and trace epidemic origins. ${ }^{63}$ Data collection can be done with the help of sensors incorporated in mobile phones, drones, robots, as well as self-sampling COVID-19 tests. The data collected via these methods are sent to a central-cloud server for analysis. The analysis generated from such a server would better equip medical providers and government agencies to respond to the COVID-19 crisis.

With these data, medical providers would be able to provide patients with more tailored online health consultations. These online services would also enable patients to receive more adequate care while simultaneously limiting their own exposure and further transmission of the virus. Government agencies, including local state health bureaus and Centers for Disease Control and Prevention (CDC), would be better able to allocate supplies, determine need for quarantine, monitor incidence, and implement emergency strategies with this information. ${ }^{63}$

Large cities are already integrating these applications to mitigate the pandemic. For example, the Shanghai Public Health Clinical Center is using body temperature sensors along with data transmission directly to nurse's station for real-time monitoring of COVID-19 patients, thus reducing the potential exposure of healthcare staff. ${ }^{28}$ Similarly, in Boston, a robot currently used to take patient interviews is about to be deployed with sensors in order to measure patients' respiratory rate and body temperature. ${ }^{8}$ In Singapore, a contact-tracing smartphone application uses wireless Bluetooth technology in order to identify people who have been in close proximity with COVID19 patients. ${ }^{5}$ Apple and Google collaborate in contact tracing and tracking applications that will become available to many countries worldwide, and is expected to dramatically accelerate the identification and notification of individuals that have been unknowingly close to COVID-19 patients. ${ }^{39}$ IoMT would not only serve to help with the current pandemic, but also could be implemented to prevent future outbreaks as well. 


\section{Community Screening}

The COVID-19 pandemic has created an unprecedented need for contact tracing, case detection, and isolation for containing. ${ }^{52}$ There have been two proposals addressing population screening for case detection: massive random screening and targeted screening. ${ }^{15}$ The latter comprises of three complementary testing strategies in order to maximize the number of infected people detected. The first strategy is to remove physician referral and cost restrictions for testing patients with symptoms suggestive of COVID$19 .{ }^{15}$ The second strategy is to select and test people in high-risk sentinel locations, like elderly in nursing homes and pregnant women presenting for delivery. ${ }^{15}$ The third strategy is to reserve a small number of tests for random screening in order to assess whether sentinel sites are efficiently detecting infected people. ${ }^{15}$ Both population screening methods have to be followed by contact tracing and isolation of people testing positive. This could bring the reproductive number (Ro) of COVID-19 to less than unity and end the repeated cycles of imposing and releasing stay-at-home restrictions. ${ }^{15}$ Aforementioned technologies could be implemented in comparing the efficacy and cost-effectiveness of the two proposals.

\section{Telemedicine}

Telemedicine enables healthcare providers to care for patients while abiding by government shelter-in-place and social distancing orders. Throughout this pandemic, online healthcare services have been utilized to provide care for patients at home with mild COVID-19 as well as provide information about the symptoms and prevention of disease to all patients. Telemedicine services have also recently expanded in developed countries for the medical management of mild non-COVID-19 related issues. ${ }^{32}$ These services range from annual follow-ups to mental health services. Mental health services in particular have increased in demand during this time as quarantine related isolation can lead to anger, frustration, fear, and increased stress. ${ }^{32,44}$ Telemedicine services are vital as they decreased the number of hospital visits and increase hospital resources, including hospital staff time. With the increased demand on hospital workers during the healthcare crisis, telemedicine helps off-load the burden on hospital workers, enabling them to focus efforts on those with more severe conditions. ${ }^{10}$ Studies on real-world data have estimated that around $60 \%$ of US hospitals have adopted telemedicine. $^{18}$ In Massachusetts, Minnesota, New Hampshire, and Wisconsin the proportion of hospitals adopting telehealth exceeds $80 \% \cdot{ }^{18}$ A study in a large healthcare system in New York has estimated that between March 2, 2020 and April 14, 2020, telemedicine visits increased from 369.1 /day to 866.8 /day (135\% increase) in urgent care and from 94.7 /day to 4209.3 /day $\left(4345 \%\right.$ increase) in non-urgent care. ${ }^{33}$

Telemedicine can also be utilized to train staff in rural and remote areas to better respond to this healthcare crisis. ${ }^{32}$ Increased capabilities of responders in rural areas helps to ensure affected patients have access to adequate care. This remote training platform can also be used to aid countries with fewer trained medical personnel.

\section{Robotics}

Robotics can be used to minimize both patient and provider COVID-19 risk. Robotic surgery provides a safer alternative in terms of COVID-19 exposure for procedures, compared to conventional laparoscopy and open surgery. If all high level precautions are taken, there is reduced number of directly exposed medical staff, as well as reduced patient hospital stay. As a result, there is a decreased patient risk of COVID19 infection and increased hospital capacity to treat COVID-19 patients. $^{27}$

Robotics can be used to perform procedures and reduce medical staff exposure. Autonomous or remotecontrolled robots can perform noncontact ultraviolet (UV) surface disinfection via intelligent navigation and identification of high-risk/high-touch areas. ${ }^{64}$ Robots can also independently collect daily temperature measurements in inpatients with COVID-19 as well the temperatures of patients at screening centers. In addition, robots can independently conduct nasopharyngeal swab testing. ${ }^{11}$ The use of robotics to perform these functions decrease required staff contact with those infected with COVID-19 patients and would also allow staff to allocate their time towards other patient care tasks. ${ }^{47}$

Finally, Unmanned Aerial Vehicles (UAV) or drones can identify potential COVID-19 carriers. UAV have the capacity to detect ground surface temperatures from a certain height above the ground and send the measured data, captured thermal images and optical images along with GPS location, to a server. This technology can be adapted to capture body temperatures, enabling us to identify patients with fever, an early symptom of COVID-19. ${ }^{1}$ Furthermore, UAV technology can be deployed to distribute medical supplies and test kit equipment in areas difficult to reach, thus reducing mortality. ${ }^{4}$

\section{$3 D$-Printing}

Three-dimensional printing is a quickly emerging field that can help in the design of medical equipment and can more readily supply needed materials at re- 
duced costs. The COVID-19 pandemic has caused a worldwide shortage of medical supplies, including the Personal Protective Equipment (PPE), necessary for the care of COVID-19 patients. ${ }^{60}$ PPE includes facemasks, face shields and goggles. This equipment helps prevent droplet exposure and further spread of the virus. Face masks need to be fitted appropriately in order to adequately prevent air and small droplets from entering around the edges of the mask. 3D laser scanning allows measurement of exact facial parameters, enabling the production of personalized masks. ${ }^{53}$ Using open-source data, face shields can also be produced with biodegradable material, allowing at-home, on-demand production. ${ }^{59}$ Utilization of $3 \mathrm{D}$ printing technology would increase access to these supplies and create more personalized equipment that can better protect medical personnel.

Three-dimensional printing technology can assist in the production of nasopharyngeal and oropharyngeal test swabs. The assistance of $3 \mathrm{D}$ printing in production of these materials would allow for widespread population testing. With increased access to testing, policy regarding carrier tracing and isolation to prevent the spread of the virus could be more conservative and effective. ${ }^{23}$

In countries impacted heavily by COVID-19 there have been shortages in key components of the respiratory support equipment. In such settings, 3D-printing has been implemented to produce venturi valves. These valves were difficult to produce given their design being subject to copyright and patent covers. In addition, 3D printing can also be utilized for production of ventilator splitters with adjustable flow control valves. The adjustable flow control valves have further increased patient access to ventilators by enabling two patients with different oxygen requirements to use the same ventilator. ${ }^{55}$

Utilization of 3D-printing can revolutionize equipment production in terms of efficiency, quantity and cost. Large academic medical centers and hospitals should work together with the 3D printing community in order meet the rising demand of medical supplies. However, safety protocols should still be followed during this time to ensure medical supplies meet regulatory standards and are safe for use.

\section{CONCLUSION}

Emerging technologies can be effectively utilized to allow the medical community to rapidly respond to the increased demands and burden of COVID-19. Technologies have been used in the study, diagnosis, and treatment of COVID-19. Recent developments have proven the collaboration between medical researchers and engineers is essential for the development of expeditious and less expensive ways of addressing the pandemic. In the context of the rapid and worldwide disease spread, open access to knowledge, tools, and technology is essential for timely response. ${ }^{6}$ Researchers and engineers must continue to collaborate and share expertise to continue to provide solutions in this time of crisis. Although swift development and application of novel technologies is required, safety monitoring should not be overlooked. Established standards regarding patient generated data, including confidentiality must be upheld. ${ }^{22}$ In addition, the safety standards for the production and distribution of supplies and services should continuously be monitored as new technologies are being used. Treatments identified using emerging technologies should also undergo standard clinical trials. The community needs to strive to uphold all these safety standards to ensure best outcomes for patients.

\section{AUTHOR CONTRIBUTIONS}

MTV, EA, and JG drafted the initial manuscript. FS, EKM, MK edited the manuscript. EM reviewed and revised the manuscript. All authors read and approved the final manuscript as submitted and agreed to be accountable for all aspects of the work in ensuring that questions related to the accuracy or integrity of any part of the work are appropriately investigated and resolved.

\section{REFERENCES}

${ }^{1}$ Abdulrazaq, A. P. D. M., N. Hazairin, S. Al-Zubaidi, S. Karim, S. Mustapha, and E. Yusuf. Toward a novel design for Coronavirus detection and diagnosis system using IoT based drone technology. Int. J. Psychosoc. Rehabil. 24:2287-2295, 2020.

${ }^{2}$ Adam, D. Special report: The simulations driving the world's response to COVID-19. Nature 580(7803):316-318, 2020.

${ }^{3}$ Alex, Z., A. Vladimir, Z. Alexander, Z. Bogdan, T. Victor, S. B. Dmitry, et al. Potential COVID-2019 3C-like protease inhibitors designed using generative deep learning approaches. ChemRxiv 2020. https://doi.org/10.13140/RG. 2.2.13846.98881

${ }^{4}$ Anggraeni, S., A. Maulidina, M. W. Dewi, S. Rahmadianti, Y. P. C. Rizky, Z. F. Arinalhaq, et al. The deployment of drones in sending drugs and patient blood samples COVID-19. BioRxiv 5(2):8, 2020.

${ }^{5}$ Baharudin, H., and L. Wong. Coronavirus: Singapore develops smartphone app for efficient contact tracing. $h$ ttps://www.straitstimes.com/singapore/coronavirus-singap ore-develops-smartphone-app-for-efficient-contact-tracing 
${ }^{6}$ Barton, C. M., M. Alberti, D. Ames, J. A. Atkinson, J. Bales, E. Burke, et al. Call for transparency of COVID-19 models. Science 368(6490):482-483, 2020.

${ }^{7}$ Bekiros, S., and D. Kouloumpou. SBDiEM: a new mathematical model of infectious disease dynamics. Chaos, Solitons Fractals 136:109828, 2020.

${ }^{8}$ Bray H. The robot will see you now A Boston hospital is using Spot, the dog-like robot of Internet fame, to screen for coronavirus. https://www.bostonglobe.com/2020/04/23 /business/robot-will-see-you-now/.

${ }^{9}$ Callaway, E. The race for coronavirus vaccines: a graphical guide. Nature 580(7805):576-577, 2020.

${ }^{10}$ Chauhan, V., S. Galwankar, B. Arquilla, M. Garg, S. D. Somma, A. El-Menyar, et al. Novel coronavirus (COVID19): leveraging telemedicine to optimize care while minimizing exposures and viral transmission. J. Emerg. Trauma Shock. 13(1):20-24, 2020.

${ }^{11}$ Chen, F. M., M. C. Feng, T. C. Chen, M. H. Hsieh, S. H. Kuo, H. L. Chang, et al. Big data integration and analytics to prevent a potential hospital outbreak of COVID-19 in Taiwan. J. Microbiol. Immunol. Infect. 2020. https://doi. org/10.1016/j.jmii.2020.04.010.

${ }^{12}$ Ciliberto, G., and L. Cardone. Boosting the arsenal against COVID-19 through computational drug repurposing. Drug Discov. Today 2020. https://doi.org/10.1016/j.drudis.2020. 04.005 .

${ }^{13}$ Dhama, K., K. Sharun, R. Tiwari, M. Dadar, Y. S. Malik, K. P. Singh, et al. COVID-19, an emerging coronavirus infection: advances and prospects in designing and developing vaccines, immunotherapeutics, and therapeutics. Hum. Vaccin. Immunother. 2020. https://doi.org/10.1080/2 1645515.2020.1735227.

${ }^{14}$ Dormont, F., R. Brusini, C. Cailleau, F. Reynaud, A. Peramo, A. Gendron, et al. Squalene-based multidrug nanoparticles for improved mitigation of uncontrolled inflammation. Sci Adv. 6(23):eaaz5466, 2020.

${ }^{15}$ Edward, H., and H. P. F. Kaplan. Logistics of Aggressive Community Screening for Coronavirus 2019, 2020. https:// jamanetwork.com/channels/health-forum/fullarticle/27656 93? resultClick $=1$.

${ }^{16}$ Gozes, O., M. Frid-Adar, H. Greenspan, P. D. Browning, H. Zhang, W. Ji, et al. Rapid AI development cycle for the coronavirus (COVID-19) pandemic: initial results for automated detection \& Patient Monitoring Using Deep Learning CT image analysis. arXiv e-prints [Internet], 1 March 2020 [arXiv:2003.05037p]. https://ui.adsabs.harvar d.edu/abs/2020arXiv200305037G.

${ }^{17}$ Han, Y., and P. Kral. Computational design of ACE2based peptide inhibitors of SARS-CoV-2. ACS Nano 14(4):5143-5147, 2020.

${ }^{18}$ Hong, Y. R., J. Lawrence, D. Williams, Jr., and I. A. Mainous. Population-level interest and Telehealth Capacity of US hospitals in response to COVID-19: cross-sectional analysis of Google Search and National Hospital Survey Data. JMIR Public Health Surveill. 6(2):e18961, 2020.

${ }^{19}$ Hosny, A., C. Parmar, J. Quackenbush, L. H. Schwartz, and H. Aerts. Artificial intelligence in radiology. Nat. Rev. Cancer 18(8):500-510, 2018.

${ }^{20} \mathrm{Hu}, \mathrm{T}$. Y., M. Frieman, and J. Wolfram. Insights from nanomedicine into chloroquine efficacy against COVID-19. Nat. Nanotechnol. 15(4):247-249, 2020.

${ }^{21} \mathrm{Hu}$, Z., Q. Ge, S. Li, L. Jin, and M. Xiong. Artificial intelligence forecasting of Covid-19 in China. arXiv eprints [Internet], 1 February 2020. [arXiv:2002.07112p]. h ttps://ui.adsabs.harvard.edu/abs/2020arXiv200207112H.
${ }^{22}$ Ienca, M., and E. Vayena. On the responsible use of digital data to tackle the COVID-19 pandemic. Nat. Med. 26(4):463-464, 2020.

${ }^{23}$ Ishack, S., and S. R. Lipner. Applications of 3D printing technology to address COVID-19 related supply shortages. Am. J. Med. 2020. https://doi.org/10.1016/j.amjmed.2020. 04.002 .

${ }^{24}$ Jiang, X., M. Coffee, A. Bari, J. Wang, X. Jiang, J. Huang, et al. Towards an artificial intelligence framework for datadriven prediction of coronavirus clinical severity. Comput. Mater. Contin. 62:537-551, 2020.

${ }^{25}$ Kelly, C. J., A. Karthikesalingam, M. Suleyman, G. Corrado, and D. King. Key challenges for delivering clinical impact with artificial intelligence. BMC Med. 17(1):195, 2019.

${ }^{26}$ Kim, Y. C., B. Dema, and A. Reyes-Sandoval. COVID-19 vaccines: breaking record times to first-in-human trials. NPJ Vaccines 5(1):34, 2020.

${ }^{27}$ Kimmig, R., R. H. M. Verheijen, M. Rudnicki, and for SERGS Council. Robot assisted surgery during the COVID-19 pandemic, especially for gynecological cancer: a statement of the Society of European Robotic Gynaecological Surgery (SERGS). J. Gynecol. Oncol. 31(3):e59, 2020.

${ }^{28}$ Koh, D. SPHCC employs IoT tech and wearable sensors to monitor COVID-19 patients. https://www.mobihealthnew s.com/news/asia-pacific/sphcc-employs-iot-tech-and-weara ble-sensors-monitor-covid-19-patients.

${ }^{29}$ Le Thanh, T., Z. Andreadakis, A. Kumar, R. Gomez Roman, S. Tollefsen, M. Saville, et al. The COVID-19 vaccine development landscape. Nat. Rev. Drug Discov. 19(5):305306,2020

${ }^{30}$ Lurie, N., M. Saville, R. Hatchett, and J. Halton. Developing Covid-19 vaccines at pandemic speed. N. Engl. J. Med. 382(21):1969-1973, 2020.

${ }^{31}$ Mahapatra, S., P. Nath, M. Chatterjee, N. Das, D. Kalita, P. Roy, et al. Repurposing therapeutics for COVID-19: rapid prediction of commercially available drugs through machine learning and docking. medRxiv 2020. https://doi. org/10.1101/2020.04.05.20054254.

${ }^{32}$ Mahmood, S., K. Hasan, M. Colder Carras, and A. Labrique. Global preparedness Against COVID-19: we must leverage the power of digital health. JMIR Public Health Surveill. 6(2):e18980, 2020.

${ }^{33}$ Mann, D. M., J. Chen, R. Chunara, P. A. Testa, and O. Nov. COVID-19 transforms health care through telemedicine: evidence from the field. J. Am. Med. Inform. Assoc. 2020. https://doi.org/10.1093/jamia/ocaa072.

${ }^{34}$ Mehta, P., D. F. McAuley, M. Brown, E. Sanchez, R. S. Tattersall, J. J. Manson, et al. COVID-19: consider cytokine storm syndromes and immunosuppression. Lancet 395(10229): 1033-1034, 2020.

${ }^{35}$ Mei, X., H.-C. Lee, K. Diao, M. Huang, B. Lin, C. Liu, et al. Artificial intelligence-enabled rapid diagnosis of patients with COVID-19. Nat. Med. 2020. https://doi.org/ 10.1038/s41591-020-0931-3.

${ }^{36}$ Merad, M., and J. C. Martin. Pathological inflammation in patients with COVID-19: a key role for monocytes and macrophages. Nat. Rev. Immunol. 20:355-362, 2020.

${ }^{37}$ Narin, A., C. Kaya, and Z. Pamuk. Automatic detection of coronavirus disease (COVID-19) using X-ray images and deep convolutional neural networks. arXiv e-prints [Internet], 1 March 2020 [arXiv:2003.10849p]. https://ui.adsabs. harvard.edu/abs/2020arXiv200310849N. 
${ }^{38}$ Naude, W. Artificial intelligence vs COVID-19: limitations, constraints and pitfalls. AI Soc. 2020. https://doi.org/10. 1007/s00146-020-00978-0.

${ }^{39}$ O'Neill, P. H. Apple and Google's covid-tracing tech has been released to 23 countries. https://www.technologyrevie w.com/2020/05/20/1002001/apple-and-googles-covid-tracin g-tech-has-been-released-to-22-countries/.

${ }^{40}$ Panovska-Griffiths, J. Can mathematical modelling solve the current Covid-19 crisis? BMC Public Health 20(1):551, 2020.

${ }^{41}$ Pardi, N., M. J. Hogan, F. W. Porter, and D. Weissman. mRNA vaccines - a new era in vaccinology. Nat. Rev. Drug Discov. 17(4):261-279, 2018.

${ }^{42}$ Peirlinck, M., F. Sahli Costabal, K. Linka, and E. Kuhl. Outbreak dynamics of COVID-19 in China and the United States. Biomech. Model. Mechanobiol. 2020. https://doi.or g/10.1007/s10237-020-01332-5.

${ }^{43}$ Pimentel, T. A., Z. Yan, S. A. Jeffers, K. V. Holmes, R. S. Hodges, and P. Burkhard. Peptide nanoparticles as novel immunogens: design and analysis of a prototypic severe acute respiratory syndrome vaccine. Chem. Biol. Drug Des. 73(1):53-61, 2009.

${ }^{44}$ Rajkumar, R. P. COVID-19 and mental health: A review of the existing literature. Asian J Psychiatr. 52:102066, 2020.

${ }^{45}$ Randhawa, G. S., M. P. M. Soltysiak, H. El Roz, C. P. E. de Souza, K. A. Hill, and L. Kari. Machine learning using intrinsic genomic signatures for rapid classification of novel pathogens: COVID-19 case study. PLoS ONE 15(4): $0232391,2020$.

${ }^{46}$ Rhodes, T., K. Lancaster, and M. Rosengarten. A model society: maths, models and expertise in viral outbreaks. Crit. Public Health. 30(3):253-256, 2020.

${ }^{47}$ Robert-Guroff, M. Replicating and non-replicating viral vectors for vaccine development. Curr. Opin. Biotechnol. 18(6):546-556, 2007.

${ }^{48}$ Sanders, J. M., M. L. Monogue, T. Z. Jodlowski, and J. B. Cutrell. Pharmacologic treatments for coronavirus disease 2019 (COVID-19): a review. JAMA 2020. https://doi.org/ 10.1001/JAMA.2020.6019.

${ }^{49}$ Wang, Y., X. Lu, Y. Zhang, X. Zhang, K. Wang, J. Liu, et al. Precise pulmonary scanning and reducing medical radiation exposure by developing a clinically applicable intelligent CT system: towards improving patient care. EBioMedicine 2020. https://doi.org/10.1016/j.ebiom.2020.1 02724 .

${ }^{50}$ Shi, F., J. Wang, J. Shi, Z. Wu, Q. Wang, Z. Tang, et al. Review of artificial intelligence techniques in imaging data acquisition, segmentation and diagnosis for COVID-19. arXiv e-prints [Internet], 1 April 2020 [arXiv:2004.02731p]. https:/ui.adsabs.harvard.edu/abs/2020arXiv200402731S.

${ }^{51}$ Singh, R. P., M. Javaid, A. Haleem, and R. Suman. Internet of things (IoT) applications to fight against COVID-19 pandemic. Diabetes Metab. Syndr. 14(4):521524, 2020.

${ }^{52}$ Stephenson, J. Report proposes COVID-19 National Surveillance Plan 2020. https://jamanetwork.com/channel s/health-forum/fullarticle/2765273.
${ }^{53}$ Swennen, G. R. J., L. Pottel, and P. E. Haers. Custommade 3D-printed face masks in case of pandemic crisis situations with a lack of commercially available FFP2/3 masks. Int. J. Oral Maxillofac. Surg. 49(5):673-677, 2020.

${ }^{54}$ Tang, Y., and S. Wang. Mathematic modeling of COVID19 in the United States. Emerg. Microbes Infect. 9(1):827829, 2020.

${ }^{55}$ Tino, R., R. Moore, S. Antoline, P. Ravi, N. Wake, C. N. Ionita, et al. COVID-19 and the role of 3D printing in medicine. 3D Print. Med. 6(1):11, 2020.

${ }^{56}$ Wang, C., W. Li, D. Drabek, N. M. A. Okba, R. van Haperen, A. D. M. E. Osterhaus, et al. A human monoclonal antibody blocking SARS-CoV-2 infection. Nat. Commun. 11(1):2251, 2020.

${ }^{57}$ Wang, C. J., C. Y. Ng, and R. H. Brook. Response to COVID-19 in Taiwan: big data analytics, new technology, and proactive testing. JAMA 2020. https://doi.org/10.1001/ jama.2020.3151.

${ }^{58}$ Watanabe, Y., J. D. Allen, D. Wrapp, J. S. McLellan, and M. Crispin. Site-specific glycan analysis of the SARS-CoV2 spike. Science 2020. https://doi.org/10.1126/science.abb 9983.

${ }^{59}$ Wesemann, C., S. Pieralli, T. Fretwurst, J. Nold, K. Nelson, R. Schmelzeisen, et al. 3-D printed protective equipment during COVID-19 Pandemic. Materials (Basel). 13(8):1997, 2020.

${ }^{60}$ who.int. Rolling updates on coronavirus disease (COVID19), 2020. https://www.who.int/emergencies/diseases/novel -coronavirus-2019/events-as-they-happen.

${ }^{61} \mathrm{Xu}, \mathrm{X} ., \mathrm{M}$. Han, T. Li, et al. Effective treatment of severe COVID-19 patients with tocilizumab. Proc. Natl. Acad. Sci. U.S.A. 117(20):10970-10975, 2020.

${ }^{62}$ Yan, L., H.-T. Zhang, Y. Xiao, M. Wang, C. Sun, J. Liang, et al. Prediction of criticality in patients with severe Covid19 infection using three clinical features: a machine learning-based prognostic model with clinical data in Wuhan. medRxiv 2020. https://doi.org/10.1101/2020.02.27.2002802 7.

${ }^{63}$ Yang, T., M. Gentile, C. F. Shen, and C. M. Cheng. Combining point-of-care diagnostics and Internet of Medical Things (IoMT) to combat the COVID-19 pandemic. Diagnostics (Basel). 10(4):224, 2020.

${ }^{64}$ Yang, G.-Z., B. Nelson, R. R. Murphy, H. Choset, H. Christensen, S. Collins, et al. Combating COVID-19-the role of robotics in managing public health and infectious diseases. Sci. Robot. 5(40):5589, 2020.

${ }^{65}$ Zheng, C., X. Deng, Q. Fu, Q. Zhou, J. Feng, H. Ma, et al. Deep learning-based detection for COVID-19 from chest CT using weak label. MedRxiv 2020. https://doi.org/10.11 $01 / 2020.03 .12 .20027185$.

Publisher's Note Springer Nature remains neutral with regard to jurisdictional claims in published maps and institutional affiliations. 